\title{
The effect of early developmental problems in infancy: perspectives and clinical implications
}

\author{
Nadia Micali ${ }^{1,2,3,4}$
}

Published online: 20 October 2017

(c) Springer-Verlag GmbH Germany 2017

In this month's issue, Guedeney et al. [1] set out to test the prospective association between infant social withdrawal and childhood IQ using a longitudinal prospective design. Longitudinal cohorts are amongst Europe's best resources for understanding prospective associations relevant to child development and child mental health [2]. The study hypothesised that social withdrawal at 1 year would be prospectively associated with cognitive development at 5-6 years. A great strength of the study was the availability of an assessment of motor and language development concurrent to the assessment of social behaviour. Main findings that higher social withdrawal at 1 year was prospectively associated with IQ at 5-6 years were not maintained when language and motor development scores at 1 year were included in the model. In fact, infants with high social withdrawal scores had much lower language development scores and somewhat lower motor development scores at 1 year compared to children with lower social withdrawal, thus showing a cross-sectional association between social withdrawal and motor/language development.

The authors speculate that (a) there might be an early influence of language and/or motor skills on social withdrawal behaviours, (b) language and/or motor difficulties might co-occur with social withdrawal behaviours, (c) the

Nadia Micali

nadia.micali@unige.ch

1 Child and Adolescent Psychiatry, Faculty of Medicine, University of Geneva, Geneva, Switzerland

2 Child and Adolescent Psychiatry Division, Dept. de l'Enfant et adolescent, HUG, Geneva, Switzerland

3 Institute of Child Health, UCL, London, UK

4 Department of Psychiatry, Icahn School of Medicine at Mount Sinai, New York, USA social withdrawal assessment scale might in fact be picking up language development as well as social behaviour, or (d) social withdrawal may disrupt the normal course of early cognitive development.

The research team has previously shown associations between social withdrawal at 1 year and behavioural problems at 3 years of life in the same cohort [3] and social withdrawal and language, social and communication problems at 2 years of age in another community sample [4].

The study in the current issue of ECAP raises interesting specific and broader perspectives. First, if social withdrawal is prospectively associated with psychopathology and concurrent language and motor developmental problems, one wonders whether what is being measured is actually social withdrawal. The assessment used in Guedeney et al. [1], the Alarm Distress Baby Scale (ADBB), assesses facial expression, eye contact, general level of activity, self-stimulating gestures, vocalizations, rapidity of response to stimulation, relationship with the observer, and attractiveness to the observer. As many of the items assessed seem to rely on early motor and language skills, it is possible that, particularly at this early age, the social withdrawal assessment might actually be measuring a broad set of developmental abnormalities, rather than specific deficits in the social domain. The lack of evidence of specificity of the ADBB in predicting specifically social problems longitudinally further corroborates this assumption.

Second, it is unclear if in the current study of Guedeney et al. [1] social withdrawal at 1 year is actually better than co-assessed language and motor development at predicting cognitive development at 5-6 years. It is likely that language, motor development, and social withdrawal might not be entirely separate constructs. Third, there is increasing evidence that early social, emotional and behavioural problems 
in infancy are predictive of a range of social, emotional and behavioural problems in childhood [5-9].

There has been a vast development in the last 15 years in the study and ability to assess early social-emotional and behavioural problems. Despite variability in reported specificity of early symptoms and signs and continuity of early social-emotional and behavioural problems $[8,9]$, their presence in infancy should alert parents and healthcare professionals. However, much of the available literature suggests that healthcare professionals may be reluctant to convey concerns about the development of infants and toddlers. A recent study showed that health nurses and parents rarely identify/report social, emotional and behavioural problems amongst infants. Identification of such problems occurred in only approximately one tenth of the children assessed as having social, emotional and behavioural concerns with a reliable and valid instrument [10]. Resistance in assessing and identifying early markers might be related to several factors, and might be rooted in genuine difficulties in assessing social, emotional and behavioural difficulties at an early age, as well as reluctance to label and 'stigmatize' children [11]. However, early detection, particularly in at-risk children, might prove to be very useful in establishing early intervention.

In summary, there is increasing evidence that early social, emotional and behavioural difficulties are broadly predictive of an array of psychological and developmental problems later in life. Disentangling whether the range of difficulties seen in social, emotional, and behavioural development are specific and track over time, or whether they index more general vulnerability remains to be established. Our ability as healthcare professionals to adequately detect them seems limited by a range of factors. Ultimately, detection is crucial for tertiary prevention and early intervention. A range of assessment instruments have been developed in recent years that specifically assess infant social, emotional and behavioural difficulties. Further testing not only of their psychometric properties but also of their acceptability to parents and healthcare professionals will aid to widen their use and availability. Further investigation of barriers and obstacles to early identification amongst parents and healthcare professionals specialized in infant development might also aid any such effort.

\section{References}

1. Guedeney A, Doukhan S, Forhan A et al (2017) To which extent social withdrawal at the age of 1 year is associated with IQ at 5-6 years old? Results of the EDEN mother-child cohort. Eur Child Adolesc Psychiatry. doi:10.1007/s00787-017-0988-9

2. Braddick F, Carral V, Jenkins R, Jané-Llopis E (2009) Child and adolescent mental health in Europe: infrastructures, policy and programmes. European Communities, Luxembourg

3. Guedeney A, Pingault JB, Thorr A et al (2014) Social withdrawal at 1 year is associated with emotional and behavioural problems at 3 and 5 years: the Eden mother-child cohort study. Eur Child Adolesc Psychiatry 23(12):1181-1188. doi:10.1007/ s00787-013-0513-8

4. Milne L et al (2009) Long term developmental impact of social withdrawal in infants. Infant Behav Dev. doi:10.1016/j. infbeh.2008.12.006

5. Bedford R, Wagner NJ, Rehder PD et al (2017) The role of infants' mother-directed gaze, maternal sensitivity, and emotion recognition in childhood callous unemotional behaviours. Eur Child AdolescPsychiatry 26(8):947-956. doi:10.1007/s00787-017-0967-1

6. Möricke E, Lappenschaar GAM, Swinkels SHN, Rommelse NNJ, Buitelaar JK (2013) Latent class analysis reveals five homogeneous behavioural and developmental profiles in a large Dutch population sample of infants aged 14-15 months. Eur Child Adolesc Psychiatry 22:103-115. doi:10.1007/s00787-012-0332-3

7. Briggs-Gowan MJ, Carter AS, Bosson-Heenan J, Guyer AE, Horwitz SM (2006) Are infant-toddler social-emotional and behavioral problems transient? J Am Acad Child Adolesc Psychiatry 45:849-858. doi:10.1097/01.chi.0000220849.48650.59

8. Elberling H, Linneberg A, Olsen EM, Houmann T, Rask CU, Goodman R, Skovgaard AM (2014) Infancy predictors of hyperkinetic and pervasive developmental disorders at ages 5-7 years: results from the Copenhagen Child Cohort CCC2000. J Child Psychol Psychiatr 55:1328-1335. doi:10.1111/jcpp.12256

9. Micali N, Rask CU, Olsen EM, Skovgaard AM (2016) Early predictors of childhood restrictive eating: a population-based study. J Dev Behav Pediatr 37(4):314-321. doi:10.1097/ DBP.0000000000000268

10. Alakortes J, Kovaniemi S, Carter AS et al (2017) Do child healthcare professionals and parents recognize social-emotional and behavioral problems in 1-year-old infants? Eur Child Adolesc Psychiatry 26(4):481-495. doi:10.1007/s00787-016-0909-3

11. Carter AS, Briggs-Gowan MJ, Davis NO (2004) Assessment of young children's social-emotional development and psychopathology: recent advances and recommendations for practice. J Child Psychol Psychiatry 45:109-134. doi:10.1046/j.0021-9630.2003.00316.x 